\title{
Self-Maintained Collaborative and Multidisciplinary System for Knowledge Management in Cardiology
}

\author{
J Fernández ${ }^{1}$, M Martínez-Selles ${ }^{2}$, CJ Correal ${ }^{1}$, MT Arredondo ${ }^{1}$, \\ GL Nicolosi ${ }^{3}$, Y Koumpouros ${ }^{3}$, E Martinelli ${ }^{3}$ \\ ${ }^{1}$ Universidad Politécnica de Madrid, Madrid, Spain \\ ${ }^{2}$ Hospital General Universitario Gregorio Marañón, Madrid, Spain \\ ${ }^{3}$ Regione Autonoma Friuli-Venezia-Giulia, Trieste, Italy
}

\begin{abstract}
Quality of care optimization in modern cardiology involves the collaboration of a great diversity of professionals that could benefit from the existence of collaborative work spaces. This paper presents Health Memory, a self-maintained collaborative and multidisciplinary system, specialized in cardiology, which enables different groups of healthcare professionals (e.g. general practitioners, cardiologists, personnel from medical companies, etc.), to share and improve knowledge management and communication. The system is based on a web portal through which professionals can have access to different services whose main functionality provided is the possibility of sending and searching for shared knowledge contributions. In this way, the system allows the creation of a virtual community in the cardiology domain which enables professionals to share and increase knowledge management.
\end{abstract}

\section{Introduction}

Medicine and healthcare in general have known an impressive growth and improvement in the last century and this acceleration has been even greater during the last decades. Medical research, clinical evidence and clinical studies have enormously increased (and will continue to increase) the medical knowledge.

However, this knowledge increase conflicts with its diffusion and usage in the usual daily medical practice. The progressive specialization of medical doctors and healthcare professionals gives way to the creation of specialized and, quite often, very technical knowledge domains dedicated to elites of specialists. This specialization makes communication and collaboration among general practitioners (GPs), medical doctors and other healthcare professionals very difficult outside these restricted circles.

Nowadays, quality of care optimization in modern cardiology requires network organization, continuous medical education, continuity of care among in- and out- of-hospital cardiologists and general practitioners, and an adequate ethical interaction with the medical industry, to assure awareness on the latest diagnosis and treatment advances. This situation involves the collaboration of a great diversity of professionals that could benefit from the existence of collaborative work spaces.

This paper presents a self-maintained collaborative and multidisciplinary work environment for the management of knowledge and competence in the cardiology domain. The Health Memory (HM) system offers a complete set of communication and documentation sharing tools that allow users to communicate and share their experiences and knowledge in a more effectively manner. Communication tools enable users to contact among them in both a synchronous and asynchronous way. On the other hand, documentation sharing tools are not only restricted to the consultation of specialized articles, which are already available in different Internet accessible data bases (e.g. PUBMED), or isolated guidelines, which only fit with generic situations but not with the more complicated and real ones. In this way, the system considers the sharing of users' experiences in terms of anonymous clinical cases.

\section{Methods}

The core of the HM system is a web portal which offers users an intuitive graphical user interface to access all the provided functionality. In the back-end, an agent platform enables the convenient processing of all the knowledge managed by the system. A newly developed ontology helps to improve the knowledge search and communication processes.

\subsection{User interface}

The graphical user interface (GUI) allows users to access four main system services: Information and Knowledge, Communication, Statistics and White Pages.

The 'Information \& Knowledge' service arises from the need of the different system users of sharing 
information that can be crucial in the improvement of the care process of cardiovascular patients. This sharing process can be realized either by the contribution of new knowledge to the system or by the search of stored knowledge that can satisfy the user's needs. In order to solve these information needs, the system considers different knowledge modalities. More specifically, the sharing of experienced based knowledge is addressed through the sharing of clinical cases among system users. Each one of these cases considers the anonymous description of a specific patient management process including the consultation reason, the patient's relevant history and the diagnosis and treatment details, and may include additional attached files such as, for example, ECG records or echocardiography images.

Depending on the modality of a knowledge contribution, an internal knowledge repository (KB) stores the contribution document or a reference to the document external location (e.g. a specialized library). Additionally, the knowledge repository also includes some characterizing metadata that enable system users to interact among them and contribute to the system maintenance. This interaction is achieved through the creation of user comments to a contribution and the ranking of both the contribution quality and its relevance to the knowledge search from which it has been obtained. Apart from the $\mathrm{KB}$, the $\mathrm{HM}$ system also considers the Internet as an important information source. This functionality is presented as an easy way to incorporate to the system KB users experience on different contents that are reachable by browsing the web and that may be helpful in the daily clinical practice.

Through the 'Information \& Knowledge' GUI, users have access to the knowledge search and submission facilities provided by the system. They are able to search for internal and external knowledge, commenting and valuating specific search results retrieved by the system, incorporate external knowledge to the internal $\mathrm{KB}$ through the inclusion of its reference (i.e. URL), or submit their specific contributions to the system.

The 'Communication' service arises from the need of promoting the participation of system users in discussions on different knowledge areas of interest, and the resolution of doubts or questions which may be derived from a specific care process. For this purpose, the communication tools primarily considered by the system are: a chat service that allows users to establish a quasiinstantaneous dialogue, a consultation forum which promotes the creation of interest groups, each one focused on a particular discipline or pathology, and a mail functionality that enables users to make use of their typical e-mail manager to asynchronously communicate among them and for teleconsultation purposes.

The 'Statistics' service allows the search of $\mathrm{KB}$ contribution attending to some statistical data such as their number of user accesses, the overall quality ranking, their author's name or their modality. Initially, the service provides access to the 10 most accessed documents within the system. Through the service GUI users can refine the search according to some parameters such as an author's name, a contribution modality or a contribution title. In this way, they are provided with the statistical profile of the KB contribution that best match their query.

Finally, the 'White Pages' service offers system users a way to obtain contact information about each other with the objective of improving the communication process among them. Contact information procurement takes place through a search process which considers the criteria detailed through the service GUI.

Apart from the services described above, the system GUI also provides users with other interesting functionality such as the possibility of defining their favourite knowledge contributions, or receiving notifications about the addition of new knowledge contributions to the system.

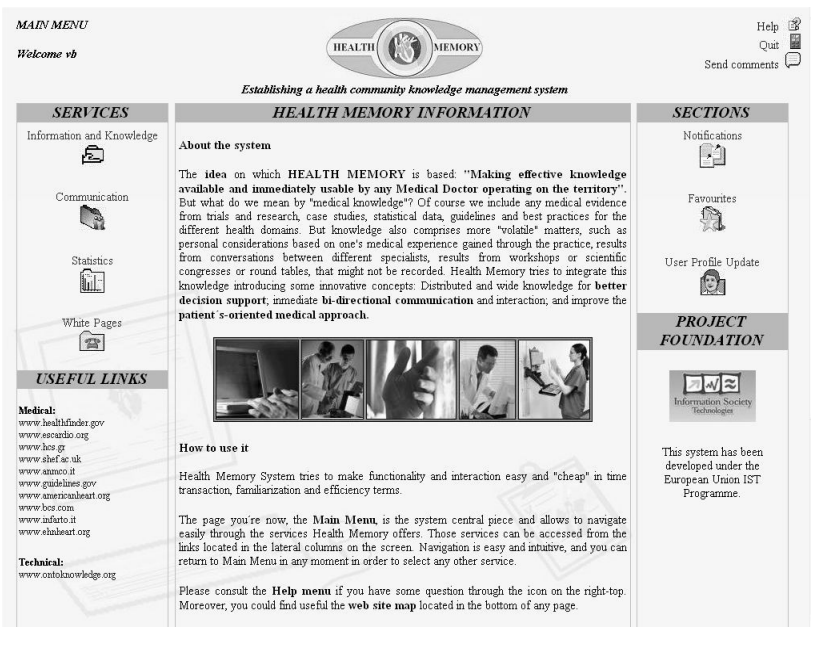

Figure 1 - Sample of the PC-based user interface.

In order to increase availability, the HM system is accessible both from a mobile and a fixed environment and the GUI is delivered in two different formats for visualization in normal PC browsers (see Figure 1) and PDA browsers respectively. In this sense, the use of the Apache Struts framework [1] in the GUI development has enabled the logical separation between the generation of the interface content, done through XML documents, and a layout adapted to the access constraints. The layout adaptation of the XML content documents has been done by the use of XSL style sheets. This approach intends to allow the future inclusion of additional GUI formats or the update of the existing ones with a minimum effort.

\subsection{Agent platform}


The agent platform is the functional core of the system. It is in charge of providing the user interface with all the functionality needed for the convenient classification, valuation and storing of the knowledge contributions submitted by system users and for the execution of the required knowledge searches.

In this way, the platform consists of the 5 specialized software agents shown in Figure 2.

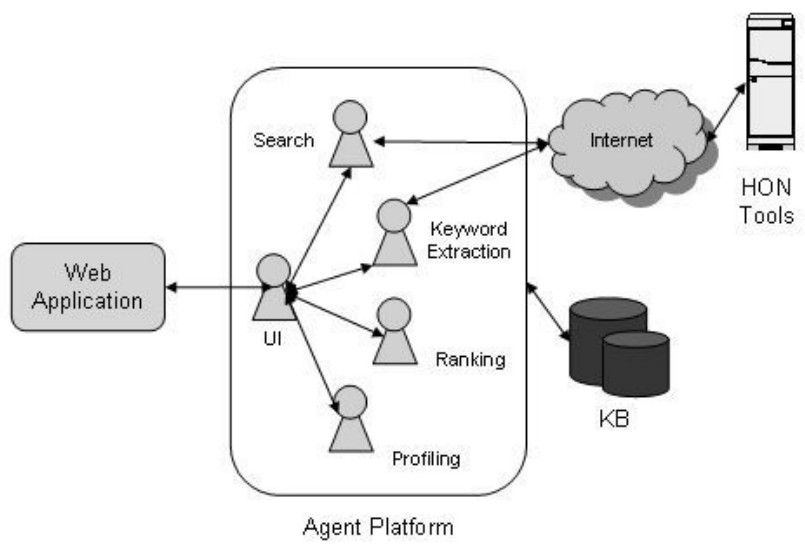

Figure 2 - Agent platform.

The UI agent acts as an intermediary among the Struts web application in charge of the provision of the system GUI and the rest of the platform. It forwards all the incoming data to a specific agent for its convenient processing and collects all the data needed for the generation of the XML content documents required by each user interaction.

The Profiling agent is in charge of managing the users' registration data. It is invoked by the UI agent for the storing of a user's data either during his registration or during a data update process. Moreover, the agent is also in charge of the verification of the user login data in order to allow him accessing some specific functionality that requires registration.

The Keyword Extraction agent is in charge of characterizing and storing each knowledge contribution submitted by a system user. This characterization is done through the extraction of the main contribution keywords. The agent checks whether these keywords are considered by the system ontology, which models the knowledge domain addressed by the system, and stores them together with the contribution in the KB.

The Ranking agent is a software entity specialized in the valuation of the knowledge considered by the system, in order to try to optimize the search. For this purpose, the agent receives through the UI agent the user subjective valuation of the search process efficiency in form of a relevance punctuation of the keywords that characterize a retrieved document according to the keywords specified by a query. In this way, the agent is able to weigh up the keywords characterization of a knowledge contribution according to the users needs. Moreover, the ranking agent also receives information about the individual impression of users on a document quality. This allows establishing a quality ranking for all the knowledge stored in the $\mathrm{KB}$, facilitating the search of relevant knowledge.

Finally, the Search agent is in charge of carrying out the search of knowledge required by a user through the corresponding query. This query is compound of keywords relevant to the target knowledge but can also consider the specification of pre-defined relations to the desired keywords. In this way, if a search query contains both keywords and relations, the agent makes use of the system ontology to look for those keywords that relate to the query specified ones through the relations. This mechanism has been made available in order to facilitate knowledge search to non-specialized users who may not be familiarized with specialized terminology proper of other cardiology sub-specialties. As it was previously mention, the search data sources used by the agent are both the KB and the Internet.

The agent platform has been implemented in JADE [2], a software framework that simplifies the implementation of multi-agent systems through a middleware that complies with the FIPA specifications for agent systems interaction. The development of the keywords extraction and search engine has considered the collaboration with the Health On the Net Foundation [3].

\subsection{The Cardiontology}

In order to empower the knowledge classification and search processes carried out by the keyword extraction and search agents, the HM system considers the use of The Cardiontology.

The Cardiontology is a domain ontology specialized in cardiology, which defines a knowledge model adapted to the needs of doctors (considering both GPs and medical doctors), pharmaceutical companies and medical technology companies. These have been defined as the user categories that are most likely to use the system.

The ontology defines the main concepts relevant to these actors within the cardiology domain and relates each other by means of relations identified by experts in the field. The Cardiontology is mainly focused in the accurate modelling of the following concept categories:

- Anatomic items related to the cardiovascular system: body regions, cardiovascular system elements (i.e. blood vessels and structural elements) and organs.

- Specialized devices used for diagnosis and therapeutic treatment of cardiac diseases, syndromes and disorders.

- Methods used for diagnosis of cardiac diseases, 
syndromes and disorders.

- Cardiovascular diseases, syndromes and disorders and their main symptoms, signs and risk factors.

- Invasive and non-invasive treatments for the adequate management of cardiovascular diseases, syndromes and disorders.

- Possible contraindications and side effects derived from the application of invasive and non-invasive treatments.

These concept categories are related to each other through the Cardiontology relations, which create direct links among concepts, enabling the ontology to automatically find "hidden" concepts from the previously detailed ones. For this purpose, the ontology primarily considers two types of relations: disease relations and treatment relations. Disease relations model the diagnosis criteria of the main cardiovascular diseases, syndromes and disorders from symptoms, signs, risk factors and diagnosing test results. On the other hand, treatment relations model the indication criteria for the treatment of cardiovascular diseases, syndromes and disorders, as well as possible contraindications or side effects derived from the application of treatments to specific clinical profiles.

The ontology development process has observed the Methonthology methodology [4] adapted to the particularities imposed by the objective knowledge domain. Concerning implementation, the ontology has been delivered as an RDF model and the conceptualization and content provision phases have considered the use of the Protégé ontology editor [5].

\section{Results}

Preliminary tests results have concluded that healthcare professionals consider the system as a useful tool for knowledge exchange. Although the system can not be viewed as a powerful search tool comparable to other specialized on-line libraries (e.g. PubMed), the possibility of exchanging comments and impressions on published knowledge (e.g. specialized articles, clinical guidelines, etc.), sharing experiences from the daily routine through the submission of clinical cases and having a shared knowledge repository for other nonpublishable knowledge, have been welcomed.

Currently, the system is in the final preparation stage for a several months testing in two hospitals: the S. Maria degli Angeli Hospital in Pordenone (Italy) and the Hospital General Universitario Gregorio Marañón in Madrid (Spain). Both experiences are focused on the assessment of system usability and users adaptation to the system services in the improvement of patients' care process. In this way, one of the main goals is the analysis of how knowledge sharing can contribute to improve medical staff experience in a patient-oriented way, and how knowledge management and IT-based tools can develop communication paths fully integrated in the structure of the Health System. For this purpose, the first test will valuate the effects and benefits on patients' care process that the use of the system will have in the interaction process among general practitioners (GPs) and hospital cardiologists. On the other hand, the second test will be more focused on the sharing of relevant experience related to the management of heart failure, acute coronary syndrome and arrhythmias among hospital and non-hospital cardiologists.

\section{Discussion and conclusions}

Modern cardiology is increasingly growing in complexity. Scientific advances lead to the development of new diagnosis, treatment and management solutions, providing healthcare professionals with a great amount of information that many times results very difficult to handle, or even remains hidden. In this context, the development of knowledge search and management systems, which facilitate communication among different cardiology areas, reveals to be an important factor.

The Health Memory system allows the creation of a virtual community in the cardiology domain which enables professionals to share and increase knowledge, as well as to improve their own specific competence, facilitating continuity of care for patients and the taking of evidence-based decisions at the point of need.

\section{Acknowledgements}

This work has been performed in the framework of the IST project Health Memory (IST-2001-38181), which is partly funded by the European Commission.

\section{References}

[1] The Apache Struts Web Application Framework. http://struts.apache.org/.

[2] Bellifemine F, Caire G, Poggi A, Rimassa JADE: A White Paper. Exp in search of innovation. Volume $4-$ n.1 March 2004. Telecom Italia Lab.

[3] Health on the Net Foundation. http://www.hon.ch/.

[4] Fernández M., Gómez-Pérez A. Juristo N. Methonthology: From Ontological Art Toward Ontological Engineering. Spring Symposium Series on Ontological Engineering. AAAI97. Stanford. USA. March 1997.

[5] Noy NF, Sintek M, Decker S, Crubezy M, Fergerson RW, Musen MA. Creating Semantic Web Contents with Protege-2000. IEEE Intelligent Systems 16(2):60-71, 2001.

Address for correspondence

Juan Fernández Ortega

E.T.S.I Telecomunicación

Ciudad Universitaria $\mathrm{s} / \mathrm{n}$.

28040 Madrid (Spain)

jfortega@gbt.tfo.upm.es 